The Canadian Journal of Higher Education, Vol. XXV-1, 1995

La revue canadienne d'enseignement supérieur, Vol. XXV-1, 1995

\title{
La situation de l'emploi chez les finissants francophones des institutions de formation à l'enseignement de l'Ontario*
}

\section{YVES HERRY, ${ }^{\dagger}$ DENIS LEVESQUE, ${ }^{\dagger}$ LAVERNE SMITH, ${ }^{\triangleleft} \&$ DAVID MARSHALL **}

\section{Résumé}

Cet article présente les résultats d'une étude portant sur la situation de l'emploi chez les finissants francophones des établissements ontariens offrant un programme de formation à l'enseignement en français. Les sujets étaient les 420 étudiantes et étudiants qui ont obtenu en 1991 un brevet d'enseignement de l'Ontario après avoir suivi un programme de formation en français. L'étude a duré deux ans. Les résultats indiquent que 63 pour 100 d'entre eux ont obtenu un poste à temps plein dans l'enseignement; 9 pour 100, un poste à temps partiel; et 15 pour 100 font de la suppléance sur appel. Selon les résultats de la deuxième année, 57 pour 100 des répondants avaient un emploi en 1991 et en 1992; 12 pour 100 ont perdu leur emploi; 6 pour 100 qui n'avaient pas d'emploi en 1991 en ont obtenu un en 1992; et 25 pour 100 n'ont enseigné ni en 1991 ni en 1992. Il y aurait donc eu une tendance à la perte d'emploi entre 1991 et 1992.

* Cet article a été rédigé dans le cadre d'un projet de recherche subventionné par le ministère de l'Éducation de l'Ontario.

$\dagger$ Université d'Ottawa

$\Delta$ Université du Nouveau-Brunswick

${ }^{* *}$ Université de Nipissing 


\section{Abstract}

The employment status of 420 Francophone students who graduated in 1991 from an Ontario French-language teacher education program was assessed, one and two years after graduation. One year after graduation, 265 graduates (63\%) held full-time teaching positions; 38 (9\%) held part-time teaching positions; and 63 (15\%) were working as supply teachers. At the two-year follow-up, 6 percent of the respondents who were not employed as teachers at the one year follow-up had signed teaching contracts; whereas 12 percent who had held a teaching position a year earlier had lost their teaching jobs. Fiftyseven percent of the respondents were found to be employed as teachers at both the one- and two-year follow-ups. Twenty-five percent of the sample had failed to secure a teaching position at either follow-up.

Un certain nombre d'études réalisées pendant les années 80 (Alberta Education, 1989; Darling-Hammond, 1984; Pajakowski, 1984; Smith, 1989) prédisaient une forte demande, voire une pénurie d'enseignantes et d'enseignants d'ici l'an 2008. En effet, les études qui ont suivi l'évolution de l'embauche des finissants en éducation de l'Ontario permettent de constater un accroissement important de la demande d'enseignants. Par exemple, seulement 38 pour 100 des finissants de 1983 obtenaient un emploi à temps plein en éducation (Atkinson \& Sussman, 1986); 50 pour 100 en 1986 (Ministère de l'Éducation de l'Ontario, 1987); 70 pour 100 en 1988 (Smith, 1989); 80 pour 100 en 1989 et 85 pour 100 en 1990 (Ministère de 1'Éducation de l'Ontario, 1991). Les données sur l'offre et la demande dans le domaine de l'enseignement faisaient croire aux chercheurs (Levesque, Poirier \& Brabant, 1991; Smith, 1989) que la croissance du taux d'embauche se poursuivrait jusqu'au début du vingt-et-unième siècle. Les chercheurs craignaient même une pénurie d'enseignants.

Ces données n'ont pas manqué d'inquiéter le ministère de l'Éducation et de la Formation de l'Ontario de même que les conseils scolaires et les facultés d'éducation, à un point tel qu'en 1990, le Ministère de l'Éducation et de la formation de l'Ontario a commandé une étude longitudinale de deux ans portant sur le taux d'embauche des finissants des établissements de formation à l'enseignement de l'Ontario (Smith, Herry, Levesque \& Marshall, 1993).

Au moment de l'appel d'offre et également pendant une partie des travaux, l'économie canadienne entrait dans une récession. N'en connaissant pas encore l'existence, la préoccupation principale du gouvernement était de savoir si les dispositions actuelles seraient suffisantes pour maintenir un équilibre entre l'offre et la demande. L'offre était principalement déterminée par le nombre de 
finissants des facultés d'éducation et la demande par les retraites et les départs, par la croissance de la population estudiantine, ainsi que par les décisions politiques comme le nombre d'élèves par classe, que le ministère de l'Éducation venait de réduire pour les classes du primaire, de même que la mise en oeuvre du jardin à temps plein, qui avant était à mi-temps.

Il va sans dire que la connaissance de l'offre et la demande en éducation est un outil de planification important pour le ministère de l'Éducation et de la Formation, de même que pour les conseils scolaires et les facultés d'éducation. Cependant, au-delà de la situation de l'emploi, certaines informations peuvent s'avérer importantes dans le cadre de la refonte actuelle du système de formation à l'enseignement. Il faut mentionner que depuis quelques années, plusieurs chercheurs et groupes de pression demandent une révision en profondeur de la formation des enseignants en Ontario (Andrews, 1984; Awender et Harte, 1986; Clifford, 1990; Fullan, Connelly \& Watson, 1990; Ontario Teachers' Federation, 1985; Pitman, 1991; Schön, 1987; Smith, 1989; Wideen \& Holborn, 1986). Plusieurs facultés d'éducation participent d'ailleurs à un effort de rationalisation et de refonte de leur programme. Une partie de la section discussion analysera les répercussions des résultats de cette étude sur certains aspects du programme de formation à l'enseignement et des politiques d'embauche des candidats par les conseils scolaires.

Cet article décrit la situation de l'emploi des 420 finissants des établissements ontariens offrant un programme de formation à l'enseignement en français. Les données couvrent une période de deux ans allant de l'obtention du brevet d'enseignement en mai 1991 jusqu'au début de l'année scolaire 19921993. Il présente les taux d'embauche des finissants de 1991 pour l'année 1991, le maintien de l'emploi pendant l'année 1992, de même qu'une analyse des variables qui influencent l'obtention d'un emploi par les finissants (Smith, Herry, Levesque \& Marshall, 1993).

\section{Méthodologie}

\section{Les sujets}

Les sujets de l'étude sont les 420 étudiantes et étudiants ( $76 \%$ de femmes) qui ont obtenu, après avoir suivi un programme de formation à l'enseignement en français, un brevet d'enseignement de l'Ontario. De ce groupe, 144 finissants proviennent de l'Université Laurentienne et 276 de l'Université d'Ottawa. Ce sont les deux seules universités ontariennes qui offrent un tel programme.

Lors de leur formation, ces étudiants devaient se spécialiser dans l'enseignement à un des trois niveaux suivants (niveaux de formation): 
primaire-moyen (maternelle à 6e année), moyen-intermédiaire (4e année à $10 \mathrm{e}$ année) et intermédiaire-supérieur (7e année à $12 \mathrm{e}$ année et Cours Préuniversitaires de l'Ontario). Les étudiants des niveaux moyen-intermédiaire et intermédiaire-supérieur devaient également se spécialiser respectivement dans une et deux didactiques (domaines de spécialisation). La répartition des finissants au sein des niveaux de formation se lit comme suit: 53 pour 100 au cycle primaire-moyen, 23 pour 100 au cycle moyen-intermédiaire et 24 pour 100 au cycle intermédiaire-supérieur. L'âge moyen des sujets est de 24 ans et 3 mois et varie entre 20 et 52 ans.

\section{Les questionnaires et le déroulement de la cueillette des données}

L'étude a nécessité trois questionnaires. Les sujets ont répondu au premier questionnaire en mars 1991 alors qu'ils complétaient leur année de formation. Il visait la collecte d'informations sur les thèmes suivants: le dossier scolaire au secondaire et au baccalauréat, les opinions et les perceptions des programmes de formation, les sentiments vis-à-vis l'enseignement, le plan de carrière, les données personnelles et démographiques. Une période d'une demi-heure pendant un cours a suffi pour remplir le questionnaire.

Le second questionnaire fut envoyé par courrier à tous les sujets en octobre 1991. L'envoi incluait une enveloppe de retour préaffranchie. Le questionnaire portait sur l'emploi actuel et sur la stratégie de recherche d'emploi.

L'envoi du troisième questionnaire a eu lieu en octobre 1992. Les sujets devaient le renvoyer par courrier à l'aide d'une enveloppe préafffranchie. Ce questionnaire incluait des questions semblables à celles du second questionnaire et une section supplémentaire portant sur leurs impressions de la première année d'enseignement (pour ceux qui avaient un emploi) et leur engagement vis-à-vis de la carrière d'enseignant.

Pour les questionnaires 2 et 3, un rappel est parvenu à tous ceux et celles qui n'avaient pas renvoyé leur questionnaire trois semaines après le premier envoi. 368 sujets $(88 \%)$ ont répondu au premier questionnaire; 304 (72\%) au second questionnaire; et $189(46 \%)$ au troisième.

\section{Les résultats}

\section{L'emploi pendant la première année (octobre 1991)}

Les résultats du tableau 1 indiquent que 86,8 pour 100 des finissantes et des finissants ont obtenu un emploi en éducation à la fin de leurs études. 72,3 pour 100 d'entre eux ont obtenu un poste à temps plein; 10,6 pour 100 , un poste à temps partiel; et 17,1 pour 100 font de la suppléance sur appel (respectivement 63 pour 100,9 pour 100 et 15 pour 100 de l'ensemble des répondants). 
Tableau 1

Nombre de finissantes et de finissants qui ont obtenu un emploi en éducation selon les types de programmes et les types de contrats (nombre de répondants = 304: $264(87 \%)$ avec emploi en éducation et $40(13 \%)$ sans emploi en éducation).

\begin{tabular}{lcccc}
\hline $\begin{array}{l}\text { Types de } \\
\text { programmes }\end{array}$ & \multicolumn{3}{c}{ Types de contrats } & Total \\
\cline { 2 - 4 } & Temps plein & Temps partiel & Suppléance \\
\hline Écoles françaises & 138 & 15 & 37 & 190 \\
Immersion & 34 & 7 & 4 & 45 \\
French Core & 17 & 5 & 4 & 26 \\
Écoles anglaises & 2 & 1 & 0 & 3 \\
Total & $191(72,3 \%)$ & $28(10,6 \%)$ & $45(17,1 \%)$ & $264(100 \%)$ \\
\hline
\end{tabular}

Le brevet d'enseignement décerné aux finissantes et aux finissants des programmes de formation à l'enseignement en français leur permet d'enseigner au sein des écoles françaises de l'Ontario. Toutefois, les étudiants peuvent, en suivant des cours post-brevet, se qualifier pour enseigner dans les écoles anglaises. Ils peuvent alors oeuvrer au sein du programme régulier anglais, du programme d'immersion française ou du programme French Core (cours de français obligatoires pour les élèves des écoles anglaises). Selon les données de cette étude, 72 pour 100 des finissants ayant obtenu un poste dans l'enseignement travaillent dans les écoles françaises; 17 pour 100 enseignent en immersion; 9,8 pour 100 enseignent le French Core; et 1,2 pour cent enseigne au sein du programme anglais régulier.

L'Ontario compte deux types de systèmes scolaires financés par la province. Le premier regroupe les écoles séparées confessionnelles et le second regroupe les écoles publiques laïques. La majorité des finissants (66\%) ayant obtenu un contrat à temps plein ou à temps partiel enseignent dans les écoles séparées catholiques. 32 pour 100 enseignent dans les écoles publiques et 2 pour 100 dans des institutions privées.

La relation entre l'obtention d'un emploi et le choix du niveau de formation par les candidats (primaire-moyen, moyen-intermédiaire, intermédiairesupérieur) a également fait l'objet d'une analyse. Elle souligne que la probabilité, pour les finissants des trois niveaux de formation, d'obtenir un 
emploi en éducation est la même, peu importe le niveau de formation choisi. Cependant, la probabilité d'enseigner au niveau pour lequel ils ont reçu une formation varie d'un groupe à l'autre. Les pourcentages se lisent comme suit: 71 pour 100 pour les finissants du primaire-moyen; 55 pour 100 pour les finissants du moyen-intermédiaire; et 87 pour 100 pour ceux de l'intermédiairesupérieur. Non seulement peu de finissants du moyen-intermédiaire enseignent à ce niveau mais très peu, à peine 21 pour 100 d'entre eux, enseignent dans leur didactique. 68 pour 100 des finissants de l'intermédiaire-supérieur sont dans cette situation.

Les finissants de l'Université Laurentienne obtiennent un taux d'embauche à temps plein plus élevé que les finissants de l'Université d'Ottawa. 77 pour 100 de tous les finissants de la Laurentienne ont obtenu un emploi à temps plein en êducation alors que c'est le cas de 58 pour 100 de ceux du programme francophone de l'Université d'Ottawa.

La grande majorité des finissants enseignent en Ontario. Seulement 3 pour 100 d'entre eux enseignent dans une autre province ou dans un autre pays. Les francophones de l'Ontario sont présents sur l'ensemble du territoire de la province. Cependant, on note de fortes concentrations dans trois régions soit le Nord (de Sudbury à la frontière nord de l'Ontario), l'Est (d'Ottawa à la frontière est de l'Ontario) et le Centre-Sud-Ouest (de Toronto à Windsor). 29 pour 100 des finissants ont été engagés dans la région Est, 30 pour 100 dans la région Centre-Sud-Ouest et 26 pour 100 dans la région Nord. La région Est engage surtout les finissants de l'Université d'Ottawa (43\% des finissants contre 3\% de ceux de l'Université Laurentienne) alors que la région Nord engagent surtout des finissants de l'Université Laurentienne (53\% des finissants contre $12 \%$ de ceux de l'Université d'Ottawa). La région Centre-Sud-Ouest engage autant de l'une que de l'autre université (25\% de la Laurentienne contre $31 \%$ d'Ottawa). Chaque institution a un territoire bien délimité et répond aux demandes spécifiques des diverses régions de l'Ontario. Ces pourcentages peuvent s'expliquer également par le degré de mobilité des étudiants dont il sera question dans une section ultérieure.

98 pour 100 de ceux qui n'ont pas eu de poste indiquent qu'ils continueront leur démarche l'année suivante pour obtenir un poste dans l'enseignement. Une quinzaine de finissants ont indiqué ne pas avoir cherché d'emploi. Les principales raisons invoquées sont le désir de poursuivre des études $(N=4)$ la maternité ou les enfants $(\mathrm{N}=3)$ et le désir de ne pas travailler à plein temps $(N=2)$. Les trois principales raisons qu'invoquent ceux qui ont cherché un emploi sans succès $(\mathrm{N}=53$ ) sont le manque de poste, l'embauche d'enseignant avec expérience et le désir de rester dans sa région. 


\section{Le maintien de l'emploi pendant la deuxième année}

Au début de la deuxième année scolaire (octobre 1992) suivant l'obtention du brevet d'enseignement, nous avons envoyé le troisième questionnaire que 46 pour 100 des finissantes et des finissants ont complété. D'après les résultats de ce questionnaire (tableau 2), 69\% des répondants ont indiqué avoir enseigné à temps plein en 1991 et $63 \%$ ont indiqué avoir enseigné à temps plein en 1992. Plus précisément, 57 pour 100 des répondants avaient un emploi en 1991 et 1992; 12 pour 100 ont perdu l'emploi qu'ils avaient en 1991; 6 pour 100 ont obtenu un emploi alors qu'ils n'enseignaient pas en 1991; et 25 pour 100 n'ont enseigné ni en 1991 ni en 1992. Il y aurait donc eu une tendance à la perte d'emploi entre 1991 et 1992.

En 1991, les finissants de l'Université Laurentienne obtenaient un taux d'embauche supérieur (77\%) à celui des finissants de l'Université d'Ottawa (58\%). Les résultats de 1992 présentent une situation légèrement différente de celle qui prévalait en 1991. Les finissants de l'Université Laurentienne ont maintenu un taux d'embauche supérieur (66\%) à celui des finissants de l'Université d'Ottawa (63\%), mais le taux d'embauche de ces derniers a augmenté de 6 pour 100 alors que celui des finissants de l'Université Laurentienne a diminué de 11 pour 100. Ce phénomène pourrait s'expliquer par la situation économique du pays en 1992. Certaines régions de l'Ontario ont été

Tableau 2

Distribution des répondants en fonction du statut d'emploi en 1991 et 1992 $(\mathrm{N}=193)$.

\begin{tabular}{cccc}
\hline Statut d'emploi dans l'enseignement & Homme & Femme & Total \\
en 1991 et 1992 & $\%$ & $\%$ & $\%$ \\
\hline
\end{tabular}

Emploi en 1991

65

55

57

et emploi en 1992

Emploi en 1991

13

12

12

et non-emploi en 1992

Non-emploi en 1991

6

6

6

et emploi en 1992

Non-emploi en 1991

16

27

25

et non-emploi en 1992 
plus affectées que d'autres par la récession. Chaque université desservant un territoire particulier, la facilité à se trouver et à conserver un emploi peut varier d'un endroit à l'autre.

Tout comme pour l'année 1991, le niveau de formation n'est pas en relation avec le taux d'embauche. La proportion de finissants qui enseignent à temps plein est la même pour les niveaux primaire-moyen, moyen-intermédiaire et intermédiaire-supérieur.

Tel que mentionné précédemment, 63 pour 100 des répondants $(\mathrm{N}=121)$ occupent un poste à plein temps dans l'enseignement. De ce nombre 54 pour 100 ont gardé le même poste que l'année précédente, 40 pour 100 ont changé de poste et 6 pour 100 n'avaient pas de poste l'année précédente. La plupart de ceux qui ont changé de poste ont changé de lieu de travail (80\%). 59 pour 100 sont à l'emploi des écoles séparées catholiques, 39 pour 100 des écoles publiques et 2 pour 100 dans les écoles privées.

\section{Les variables liées à l'emploi après l'obtention du diplôme}

Les données sur la situation de l'emploi en 1991 ont été mises en relation avec certaines informations contenues dans les questionnaires remplis par les finissants.

Parmi les variables étudiées qui étaient liées aux caractéristiques personnelles des finissants, le sexe $\left(\mathrm{Chi}^{2}=0.14\right.$, ns) et le statut marital $\left(\mathrm{Chi}^{2}=\right.$ 2.46 , ns), considérés séparément, n'influencent pas le statut d'emploi des finissants. Les hommes et les femmes de même que les personnes mariées et les célibataires ont autant de chance d'obtenir un emploi. Cependant, la combinaison de ces deux caractéristiques personnelles indique qu'une plus grande proportion de femmes célibataires que de femmes mariées obtiennent un emploi avec contrat en éducation. La situation est inverse chez les hommes; plus d'hommes mariés que d'hommes célibataires obtiennent un emploi avec contrat en éducation.

La mobilité des finissants est un facteur qui influence positivement leurs chances d'emploi en éducation ( $\mathrm{Chi}^{2}=4.1, \mathrm{p}<.05$ ). En effet, 80 pour cent des finissants qui étaient prêts à déménager pour obtenir un emploi ont obtenu un emploi alors que 69 pour cent de ceux qui ne voulaient ou ne pouvaient pas déménager ont obtenu un emploi.

L'âge des finissants est un autre facteur lié à leur statut d'emploi. La proportion de finissants qui a obtenu un emploi à temps plein ou à temps partiel diminue en fonction de l'âge (tableau 3). Le groupe de sujets âgés entre 20 et 22 ans obtient un taux d'embauche de 88 pour 100 alors que le groupe de sujets âgés de 28 ans et plus obtient un taux d'embauche de 65 pour 100. Les différences observées sont significatives $\left(\mathrm{Chi}^{2}=10.26, \mathrm{p}<.05\right)$. 
Tableau 3

Âge et statut d'emploi.

\begin{tabular}{lcc}
\hline Groupes d'âge & $\begin{array}{c}\text { Emploi à temps plein ou } \\
\text { à temps partiel }\end{array}$ & $\begin{array}{c}\text { Non-emploi } \\
\% \%\end{array}$ \\
\hline $20-22$ & 87.7 & 12.3 \\
$23-24$ & 74.7 & 25.3 \\
$25-28$ & 70.8 & 29.2 \\
$28+$ & 65.2 & .34 .8 \\
\hline \multirow{2}{*}{$2=10.26, \mathrm{p}<.05$} & & \\
\hline
\end{tabular}

Pour 100 des sujets, 10,9 ont indiqué appartenir à une minorité visible. Leur taux d'embauche est semblable à celui des sujets qui ont indiqué ne pas faire partie d'une minorité visible $\left(\mathrm{Chi}^{2}=2.90, \mathrm{~ns}\right)$.

Outre certaines informations personnelles, les questionnaires incluaient des énoncés liés à la formation académique et à l'expérience des finissants. Les résultats indiquent que le rendement académique pendant le baccalauréat $\left(\mathrm{Chi}^{2}=2.61, \mathrm{~ns}\right)$ de même que l'expérience d'enseignement $\left(\mathrm{Chi}^{2}=1.53 \mathrm{~ns}\right)$ ne sont pas liés à l'obtention d'un emploi. Toutefois, la perception du niveau de réussite des stages d'enseignement influence l'obtention d'un emploi $\left(\mathrm{Chi}^{2}=9.78, \mathrm{p}<.01\right)$. Les taux d'embauche sont respectivement de $77 \%, 74 \%$ et $54 \%$ selon que les finissants considèrent leurs stages comme excellents, bons ou satisfaisants.

Le niveau de formation (primaire-moyen, moyen intermédiaire ou intermédiaire-supérieur) n'influence pas le taux d'embauche $\left(\mathrm{Chi}^{2}=.086\right.$, ns). Toutefois, pour les cycles intermédiaire et supérieur, les résultats soulignent que certaines spécialisations sont plus en demande que d'autres. Ainsi, les diplômés ayant une spécialisation en mathématiques, en sciences (biologie, chimie, physique), en affaires et commerce et en anglais/english ont tous obtenu un emploi à temps plein. Les domaines pour lesquels la demande est moins forte sont l'histoire, la géographie, l'éducation physique et le français/french.

Dans le domaine des attitudes, l'engagement des étudiants vis-à-vis de leur programme de formation à l'enseignement de même que le degré de satisfaction de la formation reçue n'influencent pas le taux d'embauche (respectivement $\mathrm{Chi}^{2}=1.34$, ns et $\mathrm{Chi}^{2}=3.79, \mathrm{~ns}$ ). Cependant, plus les étudiants ont confiance 
de se trouver un emploi en éducation, plus cette possibilité se concrétise. Les taux d'embauche sont respectivement de 86,75 et 25 pour 100 selon qu'ils évaluaient comme excellentes, bonnes ou mauvaises leurs chances de se trouver un emploi.

Finalement, le nombre de demandes d'emploi varie de 0 à 37 pour une moyenne de 5 par étudiant et le nombre d'entrevues varie entre 0 et 15 pour une moyenne de 3 par étudiants. Les analyses de variance effectuées indiquent que le statut de l'emploi n'est pas lié au nombre de demandes d'emploi envoyées $(F(3,274)=1.78$, ns $)$ ni au nombre d'entrevues obtenues $(F(3,274)=2.04$, ns $)$.

\section{Discussion des résultats}

Cet article a présenté les résultats d'une étude de deux ans portant sur la situation de l'emploi chez les finissants francophones des établissements ontariens offrant un programme de formation à l'enseignement en français. Les sujets étaient les 420 étudiantes et étudiants qui ont obtenu en 1991 un brevet d'enseignement de l'Ontario. La première année suivant l'obtention de leur diplôme d'enseignant, 63 pour 100 d'entre eux ont obtenu un poste à temps plein dans l'enseignement; 9 pour 100 , un poste à temps partiel; et 15 pour 100 font de la suppléance sur appel. 72 pour cent des finissants ont donc signé un contrat de travail en éducation.

Ces chiffres représentent une diminution de l'emploi à temps plein par rapport aux années antérieures. Par exemple, l'année précédente (ministère de l'Éducation de l'Ontario, 1991), le taux d'embauche à temps plein atteignait 85 pour cent. En un an, ce taux aurait diminué de 22 pour 100, alors que les études (Levesque, Poirier et Brabant, 1991; Smith, 1989) prévoyaient une demande accrue, voire une pénurie d'enseignants. De plus, cette situation de l'emploi a continué de se dégrader en 1992 car l'emploi chez ces finissants pendant la deuxième année d'enseignement a diminué de 6 pour 100. Il appert que la récession qui a frappé l'économie de l'Ontario et du pays au début des années $90 \mathrm{a}$ eu un impact sur la situation de l'emploi dans l'enseignement. Les effets de cette récession combinés aux dispositions du contrat social ontarien qui vise à diminuer, entre autres, les dépenses du gouvernement devraient se poursuivre pendant encore au moins trois ans.

Toutefois, outre la situation économique difficile, d'autres variables influencent les chances d'obtenir un emploi en éducation. Ces variables sont notamment la mobilité des finissants, leur âge, leur statut marital et leur sexe.

Dans un premier temps, les finissants qui sont prêts à déménager dans une autre région sont plus nombreux à obtenir un emploi en éducation que ceux qui 
ne peuvent pas ou ne veulent pas déménager. D'autres chercheurs ont constaté l'impact de la mobilité sur le statut de l'emploi en éducation particulièrement dans les milieux où les francophones sont minoritaires (Frenette, 1988; ACELF, 1987). Ils expliquent que plusieurs finissants ont tendance à rester dans les grands centres car ceux-ci offrent plus de services aux francophones et ont des établissements d'éducation supérieure qui offrent des programmes en français (Fédération canadienne d'éducation, 1983). La plupart d'entre eux préfèrent attendre une ouverture plutôt que d'accepter un emploi dans un centre éloigné. En Ontario français, des grands centres comme Ottawa et Sudbury n'éprouvent pas de difficulté à combler leurs postes d'enseignants. Cependant, certains centres plus éloignés n'arrivent pas à combler leurs postes et doivent demander au ministère de l'Éducation et de la Formation des permissions spéciales pour engager des enseignants non qualifiés, et ce même en 1991 où un finissant sur quatre n'avait pas d'emploi.

La différence entre le taux d'embauche des finissants de l'Université Laurentienne (77\%) et celui des finissants de l'Université d'Ottawa (58\%) pourrait également être liée à leur mobilité. La grande majorité des étudiants de l'Université d'Ottawa viennent de l'Est de la province avec une forte concentration dans la région urbaine d'Ottawa-Carleton alors que ceux de 1'Université Laurentienne viennent surtout de localités éparpillées sur l'immense territoire du Nord de l'Ontario. Plusieurs de ces derniers veulent retourner dans leur région ou sont prêts à s'installer dans une autre région. Les finissants d'Ottawa sont concentrés sur un territoire plus petit et sont moins enclins à déménager dans une autre région.

L'âge des finissants est un autre facteur lié à leur statut d'emploi. Les pourcentages de finissants qui ont obtenu un emploi à temps plein ou à temps partiel diminuent en fonction de l'âge. Levesque et Reid $(1987,1989)$ et le Ministère de l'Éducation du Québec (1990) ont obtenu des résultats semblables et expliquent que le travail du conjoint ou de la conjointe, la famille en général et l'éducation des enfants empêchent les étudiants plus âgés qui retournent aux études ou qui changent de carrière d'accepter un emploi dans une autre région.

Cette explication pourrait s'appliquer aux femmes car les résultats de la présente étude indiquent que plus de femmes célibataires que de femmes mariées ou vivant en union de fait obtiennent un emploi avec contrat en éducation. L'hypothèse voudrait que les femmes célibataires ayant moins d'attaches soient plus enclines à déménager. Cependant, cette hypothèse ne tiendrait pas pour les hommes, car une plus grande proportion d'hommes mariés que d'hommes célibataires obtiennent un emploi en éducation. La mobilité seule ne suffirait donc pas à expliquer ce phénomène. D'autres variables comme 
la personnalité des finissants ou les critères de sélection des conseils scolaires pourraient jouer un rôle important dans l'obtention d'un emploi.

Les résultats de cette étude soulèvent un certain nombre de questions qui ont trait au programme de formation à l'enseignement et aux politiques d'embauche des candidats par les conseils scolaires. Les résultats les plus préoccupants sont ceux concernant la spécialisation des futurs enseignants tant pour les trois niveaux de formation (primaire-moyen, moyen-intermédiaire et intermédiaire-supérieur) que pour les domaines de spécialisation (spécialistes dans une didactique) pour les étudiants de l'intermédiaire et du secondaire. En effet, près du tiers des finisssants du primaire-moyen et la moitié des finissants du moyen-intermédiaire enseignent à un autre niveau que celui pour lequel ils ont reçu leur formation. Seuls les étudiants du secondaire échappent à cette règle: 90 pour 100 d'entre eux enseignent à l'intermédiaire-supérieur.

On pourrait mettre également en doute la pertinence de la spécialisation dans l'enseignement d'une ou de deux didactiques à l'intermédiaire-supérieur et plus particulièrement au moyen-intermédiaire, puisque les chiffres indiquent qu'un finissant sur cinq de l'intermédiaire-supérieur et sept finissants sur dix du moyen-intermédiaire n'enseignent pas dans leur didactique. En se fiant aux statistiques de l'embauche, il est surtout difficile de justifier le maintien du niveau de formation au cycle moyen-intermédiaire et encore plus celui des didactiques pour ce niveau.

La refonte du programme scolaire de l'Ontario constitue un autre élément qui milite contre la spécialisation des futurs enseignants pour le niveau moyenintermédiaire. Le document intitulé Le programme d'études commun: de la première à la neuvième année (ministère de l'Éducation et de la Formation de l'Ontario, 1993) préconise un décloisonnement des matières et une intégration des apprentissages. Ainsi des domaines comme la géographie, l'histoire, les valeurs humaines, la santé et l'éducation physique n'existent plus en soi et font partie d'un seul domaine appelé Individu et société. Il est clair que la tendance est à une formation générale plutôt que spécialisée.

Il faut préciser que le niveau de formation (moyen-intermédiaire) visait à desservir les écoles intermédiaires de l'Ontario. Ce sont des écoles qui regroupent exclusivement des élèves de $7 \mathrm{e}$ et $8 \mathrm{e}$ année. Toutefois, les écoles françaises de l'Ontario comptent très peu d'écoles intermédiaires. Le nombre insuffisant d'élèves francophones ne permet pas la mise sur pied de telles écoles sauf dans les grands centres. La plupart des élèves de $7 \mathrm{e}$ et $8 \mathrm{e}$ années fréquentent alors les écoles élémentaires.

Au sein du programme de formation initiale des enseignants, le niveau de formation moyen-intermédiaire regroupe, en théorie, des étudiants qui ne 
veulent enseigner ni au primaire ni au secondaire mais qui désirent enseigner en $7 \mathrm{e}$ et $8 \mathrm{e}$ année. En pratique, il inclut surtout des candidats qui désirent enseigner au secondaire mais qui n'ont pas les cours requis pour être admis au niveau de formation intermédiaire-supérieur. La suppression du niveau de formation moyen-intermédiaire forcerait les candidats à opter pour le primaire ou à suivre des cours additionnels qui leur permettraient de s'inscrire au niveau intermédiaire-supérieur.

Les statistiques de cette étude ont également des répercussions particulières sur la formation des enseignants dans un contexte francophone minoritaire. Par exemple, elles indiquent que les régions ont des préférences pour les candidats de certaines institutions. Les conseils scolaires de l'est de la province engagent surtout des finissants de l'Université d'Ottawa alors que ceux du Nord engagent surtout des finissants de l'Université Laurentienne. Cela peut évidemment s'expliquer par le niveau de mobilité des finissants, mais il n'est pas rare que certains conseils scolaires, surtout ceux hors des grands centres et dans des milieux francophones très minoritaires, préfèrent engager des finissants de l'Université Laurentienne, car plusieurs proviennent de ce type de milieu. Ils pourraient estimer que ces étudiants ont une meilleure compréhension de la vie en milieu minoritaire et une plus grande chance de s'adapter à leur milieu que ceux de l'Est de la province. Selon ce raisonnement, la capacité des candidats à s'adapter et à enseigner dans un milieu minoritaire proviendrait surtout de leur expérience de vie plutôt que de la formation reçue. Les programmes de formation à l'enseignement francophones ontariens ne devraient-ils pas assurer une formation dans ce domaine?

De plus, l'histoire des écoles françaises de l'Ontario est très liée à la religion catholique. Les étudiants qui veulent enseigner dans les écoles catholiques doivent avoir suivi un cours en enseignement religieux alors que les écoles publiques n'ont pas la même exigence pour le cours d'enseignement des valeurs humaines. À cause de cette exigence, plus de 90 pour 100 des étudiants s'inscrivent au cours d'enseignement religieux. Cependant, depuis une quinzaine d'années les écoles publiques, surtout dans les centres urbains, ont pris de l'ampleur et les résultats de l'étude indiquent qu'entre 30 et 40 pour 100 des finissants enseignent dans une école publique, plurireligieuse et pluriethnique. Plusieurs de ces étudiants ne reçoivent donc pas toute la formation dont ils ont besoin pour oeuvrer dans ces écoles.

Toujours dans le contexte d'une réforme de la formation des enseignants, plusieurs chercheurs en éducation misent de plus en plus sur l'induction, processus graduel de prise en charge d'une classe, échelonné sur un certain 
nombre d'années, qui permet au nouvel enseignant de mieux intégrer les diverses fonctions liées à sa profession. L'induction devrait atténuer ce que plusieurs auteurs appellent le « choc de la réalité » (Huling-Austin, 1992; Johnson, Ratsoy, Holdaway \& Friesen, 1993; Veenman, 1984). Ce dernier correspond à une prise de conscience, parfois aiguë, qui se produit lors du passage entre le statut d'étudiant encadré au sein d'un programme de formation à celui d'enseignante ou d'enseignant, seul responsable de sa classe. L'induction serait une période de stabilité au niveau de l'emploi qui permettrait au nouvel enseignant de se concentrer sur l'apprentissage des nombreuses facettes de sa profession. Cependant, les résultats de l'étude indiquent que seule la moitié (54 pour 100) de ceux qui occupent un poste à plein temps dans l'enseignement ont gardé le même poste que l'année précédente. Il est difficile de concevoir un mode de prestation de cette induction dans de telles conditions. De plus, il est paradoxal de constater qu'à cause du mode d'affectation des postes, les nouveaux enseignants sont les plus susceptibles de changer de poste, alors que ce sont eux qui auraient le plus besoin de stabilité pour maîtriser le choc de la réalité et s'adapter dans des conditions optimales au monde de l'enseignement.

Certains résultats soulèvent des questions concernant les processus de sélection utilisés par les employeurs lors de l'embauche de nouveaux enseignants, car certaines variables que l'on pourrait considérer comme importantes dans un processus de sélection n'ont pas d'influence marquée sur l'embauche. C'est le cas notamment des notes obtenues pendant le baccalauréat et de l'expérience d'enseignement antérieure à la formation. De plus, les employeurs ont à leur disposition les évaluations de stages et les notes des cours suivis par les candidats lors de la formation à l'enseignement. Il semble que ces évaluations, relativement fortes et peu discriminantes, ne permettent pas ou peu aux conseils scolaires de les utiliser pour des fins de sélection, sauf pour retenir ou rejeter les cas extrêmes. Leur choix reposerait donc principalement sur les entrevues ou sur les références du candidat.

Il est tout de même surprenant que l'expérience d'enseignement des futurs enseignants n'ait pas d'effet sur l'embauche, alors qu'elle constitue un des critères d'admission au programme de formation à l'enseignement à l'Université d'Ottawa. Par contre, des variables comme l'âge et une combinaison du sexe et du statut marital semblent influencer, plus que toute autre variable évaluée, le taux d'embauche des finissants. Cette influence peut s'expliquer par la mobilité des candidats mais il se pourrait que le processus de sélection des conseils scolaires contribuent à l'influence de ces variables sur le taux d'embauche. 
Finalement, la perception par le candidat du niveau de réussite des stages et la confiance de se trouver un emploi en éducation influencent également le taux d'embauche. Plus celles-ci sont élevées, plus la possibilité d'obtenir un emploi se concrétise. Puisque l'embauche n'est pas liée au nombre de demandes d'emploi envoyées ni au nombre d'entrevues réalisées, doit-on y voir un effet pygmalion qui fait en sorte que cette forte confiance en soi transparaît en entrevue et influence la décision des employeurs? Cette étude ne peut fournir de réponses à ces questions car elle ne portait sur les processus d'embauche utilisés par les conseils scolaires. D'autres études seraient nécessaires pour mieux comprendre le phénomène.

\section{Références}

Alberta Education. (1989). Update on teacher supply and demand in Alberta. Edmonton: Planning an Information Services Division.

Andrews, J. (1984). Alternative futures for faculties of education. Canadian Journal of Education, 9, 261-275.

Association canadienne française d'éducation de langue française (1987). Rapport $d u$ comité national pour la formation des mâtres. Sillery: ACELF.

Atkinson, T., \& Sussman S. (1986). Alternative futures for Ontario schools and some implications for performance appraisal systems. Toronto: Ministry of Education, Ontario.

Awender, M., \& Harte, A. (1986). Teacher education: What's wrong with current practice. Education Canada, 26, 24-26.

Clifford, F. (1990). The Teacher Education Council, Ontario: six months later. In R. Van Fossen (Ed.), To make a difference: Teacher education for the 1990s. Toronto: Council of Ontario Universities.

Darling-Hammond, L. (1984). Beyond the commission reports: The coming crisis in teaching. Santa Monica: Rand Corporation.

Fédération canadienne d'éducation (1983). Enseignants francophones, enseignants isolés. Ottawa: FCE.

Frenette, N. (1988). Rapport de l'enquête sur les besoins des enseignants francoontariens en matière de formation initiale et continue. Toronto: ministère de l'Éducation.

Fullan, M., Connelly, M., \& Watson, N. (1990). Teacher education in Ontario: Current practice and options for the future. Toronto: Ministries of Education and Colleges and Universities.

Huling-Austin, L. (1992). Research on learning to teach: Implications for teacher induction and mentoring programs. Journal of Teacher Education, 43(3), 173-180.

Johnson, N.A., Ratsoy, E.W., Holdaway, E.A., \& Friesen, D. (1993). The induction of Teachers: A Major Internship Program. Journal of Teacher Education, 44, 296-304. Levesque, D., \& Reid G. (1987). Rapport d'embauche 1987: Formation à l'enseignement. Ottawa: Faculté d'éducation. 
Levesque, D., \& Reid G. (1989). Rapport d'embauche 1989: Formation à l'enseignement. Ottawa: Faculté d'éducation.

Levesque, D., Poirier, P., \& Brabant, M. (1991). Étude de l'offre et de la demande d'enseignants francophones en Ontario. Toronto: ministère de l'Éducation.

Ministère de l'Éducation de l'Ontario (1987). Education Statistics, Ontario. Toronto: Ministère de l'Éducation.

Ministère de l'Éducation de l'Ontario (1991). Education Statistics, Ontario. Toronto: Ministère de l'Éducation.

Ministère de l'Éducation de l'Ontario (1993). Le programme d'études commun: de la première à la neuvième année. Toronto: Ministère de l'Éducation.

Ministère de l'Éducation du Québec (1990). Les besoins de recrutement de personnel enseignant à temps plein par les commissions scolaires de 1989 à 2011. Québec: Direction générale de la recherche et du développement.

Ontario Teachers' Federation (1985). The clouded crystal. Submission to the Cabinet. Toronto: Ontario Teachers' Federation.

Pajakowski, M. (1984). An annotated bibliography of the Literature dealing with educational trends and the effect this has on the teaching workforce. Indianapolis: University of Indiana.

Pitman, W. (1991). Training teachers for global education: An educator's personal view. Perspective, 2, 1.

Schön, D. (1987). Educating the reflective practitioner: Towards a new design for teaching and learning in the professions. San Francisco: Jossey-Bass.

Smith, L. (1989). Perspectives sur l'offre et la demande d'enseignants en Ontario, 19882008. Toronto: Imprimeur de la Reine.

Smith, L., Herry, Y., Levesque, D., \& Marshall D. (1993). On becoming a teacher: A longitudinal study. Toronto: ministère de l'Éducation.

Veenman, S. (1984). Perceived problems of beginning teachers. Review of educational research, 54, 143-178.

Wideen, M., \& Holborn, P. (1986). Change and survival in faculties of education. Interchange, 17, 33-47. 